\title{
Editorial
}

\section{Drug delivery research in Europe}

Tremendous advances have been made in the drug delivery field in the past few decades. In the early days of drug delivery research, i.e., in the 1950-1980s, the field was dominated by scientists working on macro- and microscopic depot devices, which aimed to enable sustained and/or improved drug delivery upon implantation, ingestion or topical administration. From the 1980s onwards, based on the magic bullet concept (proposed by Paul Ehrlich in 1905) and on the Enhanced Permeability and Retention effect (first described by Maeda and colleagues in 1986), advances in chemical and pharmaceutical engineering, in bio- and nanotechnology, and in genetic and molecular medicine rapidly fueled growth in drug delivery systems for systemic drug targeting, aiming to more efficiently and more specifically target i.v. administered (chemo-) therapeutic agents to pathological sites, to thereby improve the balance between their efficacy and their toxicity.

The drug delivery field has grown exponentially in the last 20 years. This is exemplified by Fig. 1, which shows that up until 1990, only about a 1000 "drug delivery" papers could be retrieved in Web of Science. Ever since, however, the field has started to grow at an everincreasing pace, and especially from 2005 onwards, a huge increase in the number of papers published per year can be observed. The total number of drug delivery papers published worldwide currently is $\sim 99,000$, and it therefore is highly likely that somewhere in 2012, the magical 100,000 mark will be surpassed. Fig. 1 furthermore exemplifies that when it comes to the number of publications per continent, there is not much of a difference between North America, Europe and Asia. In all three continents, thousands of scientists and hundreds of research groups are working on the development of novel systems and strategies for drug delivery and drug targeting, and this is currently resulting in several thousands of papers published per continent per year.

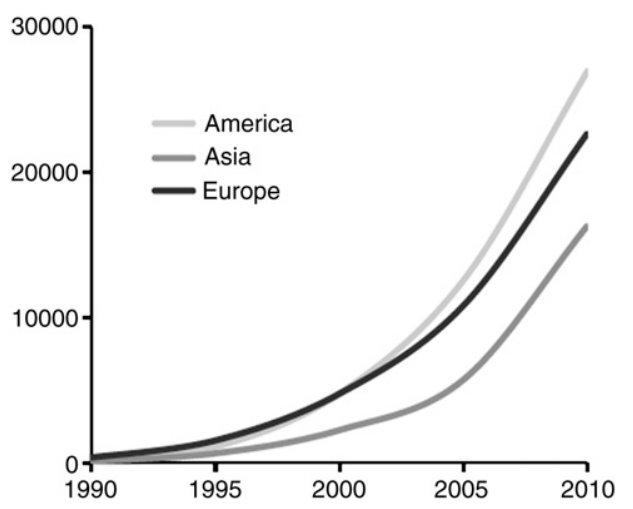

Fig. 1. Number of publications on "Drug delivery" listed in Web of Science.
Another aspect of drug delivery research that has tremendously expanded over the years is the number of applications for which delivery systems and targeting strategies are being developed. In the early days, they were almost exclusively focused on genetic disorders and cancer, but in recent years, also inflammatory, metabolic and organ-specific disorders have been attracting significant attention. Furthermore, as a result of advances in nanotechnology and in chemical and pharmaceutical engineering, ever more carrier materials are nowadays being employed for drug delivery purposes, including besides liposomes, polymers, proteins and micelles, e.g. also various different types of nanoparticles, nanotubes, nanorods, nanobullets, nanospheres and nanoshells.

Ever since its inception in 1984, the Journal of Controlled Release (JCR) has been a leading forum for showcasing advances in the drug delivery field. Initially focusing primarily on sustained drug release from implantable or injectable depot devices, the scope of the Journal has expanded considerably over the years. JCR now covers not only drug delivery systems, drug release mechanisms and drug targeting strategies, but also biological properties of drugs, bioactive polymers and hydrogels, tissue engineering, molecular and functional imaging, gene therapy and nanomedicine.

In the present issue, in an attempt to comprehensively summarize the progress made in the drug delivery field in the last couple of years, 50 leading European reseachers and/or research teams describe the advances made in their respective areas of expertise. The areas covered are as diverse as the drug delivery field itself, addressing different carrier materials, different targeting strategies and different diseases, as well as biotechnological, nanotoxicological, immunological, methodological and economical aspects. This issue therefore serves as a European benchmark for furthering, fostering and fine-tuning drug delivery research worldwide.

Twan Lammers RWTH Aachen University and Helmholtz Institute for Biomedical Engineering, Department of Experimental Molecular Imaging, Aachen, Germany

University of Twente and MIRA Institute for Biomedical Technology and Technical Medicine, Department of Targeted Therapeutics, Enschede,

The Netherlands

Utrecht University and Utrecht Institute for Pharmaceutical Sciences, Department of Pharmaceutics, Utrecht, The Netherlands E-mail address: tlammers@ukaachen.de. 